\title{
NOTAS SOBRE EL VALOR EDUCATIVO DE LA GEOGRAFÍA DE LA POBLACIÓN*
}

\author{
Vicente Gozálvez Pérez
}

\section{RESUMEN}

Las cuestiones relativas a la población preocupan cada vez más a las diversas sociedades del planeta, aunque con ópticas o intereses que incluso pueden ser contrapuestos según espacios o sociedades, al mismo tiempo que son patentes las interdependencias geodemográficoas entre naciones. Sin embargo, existe evidente disociación entre la importancia y utilidad que actualmente da la sociedad a los temas geodemográficos, y el escaso desarrollo académico —en sus diversos niveles — de la demografía geográfica; es preciso desarrollar más esta última, para que las cuestiones geodemográficas puedan ser juzgadas y tratadas con más conocimiento de causa o con más efectividad. Así, la geodemografía (distribución espacial de los contingentes, de sus dinámicas naturales y migratorias, así como de sus estructuras demográficas, económicas y socio-culturales) sin duda puede y debe ser utilizada como instrumento eficaz y útil tanto por los que tienen responsabilidades en la ordenación y gestión del territorio, como por el ciudadano - en sus diversas funciones o con sus diversas formaciones- que la debe usar para comprender mejor las características y los comportamientos geodemográficos de las distintas sociedades.

Palabras clave: geografía de la población, instrucción y educación, ordenación del territorio.

\section{RÉSUMÉ}

Les questions concernants la population intéressent de plus en plus les diverses sociétés du monde, mais avec des visions ou des intérêts parfois opposés suivant les espaces ou les sociétés, tandis que sont evidentes les interdépendances geodémographiques entre les nations. Cependant, il existe une évidente dissociation entre l'importance et l'utilité qu'aujord'hui donne la société aux sujets géodémographiques, et l'insuffisant développement académique —à ses divers niveaux — de la démographie géographique;

* Este texto resume la Conferencia de clausura de las IV Jornadas de Didáctica de la Geografía, «Educación y Geografía», celebradas en la Universidad de Alicante durante el 27 y 28 de noviembre de 1998. 
il est necessaire de développer plus celle-ci, pour que les questions géodémographiques puissent être jugées et traitées avec plus connaissance ou avec plus efficacité. Ainsi, la géodémographie (distribution spaciale de la population, de ses dynamiques naturelles et migratoires, ainsi que de ses structures démographiques, économiques et socioculturelles) peut et doit sans doute être utilisée comme instrument efficace et utile aussi bien par ceux qui ont des responsabilités dans l'aménagement et la gestion du territoire, comme par les citoyens - dans leurs diverses fonctions ou avec leurs diverses formations-qui doivent l'employer pour comprendre mieux les caracteristiques et les comportements géodémographiques des différentes sociétés.

Mots clés: géographie de la population, instruction et éducation, aménagement du territoire.

\section{Introducción}

Las cuestiones relativas a la población es evidente que preocupan cada vez más a las diversas sociedades del planeta, aunque, obviamente, con ópticas, intereses o problemáticas que incluso pueden ser contrapuestas según espacios, tiempos o sociedades. Las poblaciones de Europa y de África ilustran bien lo indicado, pues actualmente tienen características que las muestran como antítesis demográficas pese a su cercanía geográfica. Así, las poblaciones de ambos continentes quedan definidas, respectivamente, por fecundidades bajas y altas, por vejez y juventud demográficas, por inmigración y emigración internacionales, por crecimiento natural casi cero o negativo y tasas aún «explosivas», etc.

En nuestra actual era de la globalización, el conocimiento de estas situaciones geodemográficas contrapuestas interesa a todo tipo de sociedades humanas, pues son patentes las interdependencias geodemográficas entre naciones, que sin duda crecerán en el futuro próximo; la evolución reciente de las migraciones internacionales que experimenta Europa, es tal vez el ejemplo más tratado en los medios de comunicación - y por ello más próximo al ciudadano- sobre dichas interrelaciones geodemográficas internacionales.

Sin embargo, mientras se generalizan los protagonismos concedidos a las cuestiones geodemográficas, en España aún se cumple casi literalmente la famosa frase de Alfred Sauvy cuando calificaba a la demografía como «ciencia sin maestros ni alumnos» ${ }^{1}$, es decir, en nuestro caso aún existe una evidente disociación, o al menos una gran distancia, entre, por un lado, la aparente importancia y utilidad percibidas por la sociedad respecto a los temas geodemográficos, y, por otro, el escaso desarrollo de la vertiente académica — docente y discente - de la demografía geográfica. Así, sin duda es necesario corregir las actuales deficiencias formativas e informativas sobre geodemografía, para que tanto los grandes temas sobre las poblaciones que hoy suscitan graves y preocupantes debates, como también los «pequeños» temas geodemográficos — por la reducida escala espacial a que afectan o por su menor atención social一, puedan ser enunciados, juzgados y tratados por los diversos miembros de nuestra sociedad, con más conocimiento de causa o con más efectividad.

1 LEVY, M.L., «Spécifité de la démographie: l'analyse longitudinale», Population et Societés, Paris, I.N.E.D., num. 284, nov. 1993. 
En la actualidad la definición y los contenidos de la geografía de la población merecen consenso de fondo entre los autores ${ }^{2}$, es decir, el tratamiento espacial de la población, tanto en sus cifras o contingentes, como en sus dinámicas naturales, en sus movilidades y en sus estructuras demográficas, económicas y socioculturales. Por otra parte, la información geodemográfica, convenientemente elaborada, tiene aplicaciones o utilizaciones mucho más amplias, pues se sitúa, o debe hacerlo, en el centro de las decisiones de los agentes sociales, o dicho de otra forma, las interacciones entre la geodemografía y la ordenación del territorio son múltiples. El conocimiento de la geodemografía sin duda ayuda eficazmente a comprender los funcionamientos y las características de las sociedades, y precisamente por ello hay que concebir y enfocar la geodemografía, y sus fuentes de información, de modo que sean útiles tanto al planificador como a los ciudadanos en sus diversas funciones o con sus diversas formaciones. Así, la geodemografía puede y debe ser concebida, al menos en parte, como una ciencia política en el sentido pleno del término, debe contribuir a la mejor gestión de los asuntos públicos.

Mi intervención, forzosamente breve como corresponde a la clausura de estas IV Jornadas de Didáctica de la Geografía se limitará a ofrecerles algunas reflexiones o inquietudes sobre la geografía de la población, mías o elaboradas por otros, que juzgo de interés, dentro de la temática general de estas Jornadas, «Educación y Geografía».

\section{Geografía de la población: de la educación a la praxis}

Las insuficiencias formativas e informativas sobre geografía demográfica, en España con frecuencia afectan no sólo al ciudadano más joven, es decir al estudiante, sino también a aquel que ha asumido responsabilidades en política o en ordenación del territorio, e incluso al docente. Esta situación en parte puede tener una «justificación oficial», debido a la juventud universitaria de las escasas disciplinas que se ocupan de la población, pues en el caso de la geografía de la población, ésta se incorpora muy tardíamente a nuestros planes de estudio: en los de 1973 la geografía de la población, aunque con implantación general, con frecuencia lo fue con carácter optativo, y sólo en el nuevo plan de Licenciado en Geografía de 1993 se ha generalizado su docencia obligatoria y con duración anual.

Por otra parte, la disociación entre la importancia y el uso de la información geodemográfica, no parece ser privativa de España, pues carencias semejantes son denunciadas desde antiguo y en la actualidad incluso en países como Francia, donde las materias relativas a la población están mucho más cuidadas y consolidadas tanto en la docencia de grado medio y superior, como en la investigación. Por otra parte, la disociación que comentamos, también puede ser fruto de falta de comunicación, tal como ya denunciara hace décadas Alfred Sauvy, fundador y primer director del Instituto de Estudios Demográficos de Francia (1945): el papel del experto es comprender, después informar, «ciencia pura, ciencia aplicada, la demografía no puede escapar a esta doble tarea, ...» ${ }^{3}$. Persuadido Sauvy de que el riesgo del poder es ignorar el saber, en nuestro caso las cuestiones relativas a la población, el investigador debe hacer conocer los hechos a los responsables políticos así como a un público amplio, de modo que la tarea educativa en cuestiones de

2 GOZÁLVEZ PÉREZ, V., «Las nuevas perspectivas del análisis geográfico de la población», Iber, Didáctica de las Ciencias Sociales, Geografía e Historia, núm. 16, Barcelona, Edit. Graó, abril 1998, pp. 33-43.

3 VÉRON, J., «Alfred Sauvy aurait cent ans», Population et Societés, Paris, I.N.E.D., num. 339, oct. 1998. 
población no debe ser menos importante que la investigadora. En 1958, en su libro De Malthus a Mao Tse-Toung, Sauvy señalaba un desideratum educativo que en modo alguno se ha conseguido después de cuarenta años: hemos de procurar que las informaciones sobre población transmitidas al público tiendan a instruirle y no a sugestionarle. Los medios de comunicación están especialmente ávidos de titulares impactantes — tentación que no es ajena a docentes e investigadores-, pero las consecuencias de los fenómenos demográficos se inscriben y se manifiestan en las sociedades después de un tiempo más o menos prolongado, con lo que también hay que contar con las modificaciones que razonablemente puedan ir surgiendo durante estos periodos; así, los titulares pueden llamar la atención, pero no son suficientes para instruir.

Después de décadas, en 1995 el actual director del I.N.E.D., Patrick Festy, en una entrevista titulada «iY si los poderes públicos escucharan un poco más a los demógrafos?», continúa quejándose de que los poderes públicos «muestran una curiosidad más bien escasa respecto a los problemas demográficos $»^{4}$. Y es que, como es muy frecuente, el investigador y el político, tanto en su trabajo y cometidos diarios, como en las escalas temporales que utilizan, tienen «naturalezas» o lógicas diferentes.

Así, como señala Leon Gani ${ }^{5}$, en la actualidad es necesaria una «alfabetización demográfica», o geodemográfica que diríamos los geógrafos, aunque los demógrafos también se acercan cada vez más a la demografía espacial, persuadidos de la necesidad de desarrollar la demografía aplicada en sus diferenciaciones territoriales. Gani (Université Paris-V) califica de «muy deficiente» la educación de los jóvenes europeos en materia de población, toda vez que estos conocimientos son necesarios para comprender la vida social, tanto en la que ellos mismos se desenvuelven, como la que afecta a las sociedades de los países menos desarrollados. Los conceptos e interpretación de hechos como las transiciones demográficas o la lectura actual y prospectiva de los perfiles de las pirámides de edades, es algo básico para interpretar, entre otros, los balances del movimiento natural, los temas de empleo, de necesidades de infraestructuras o de servicios para los diversos grupos de edad, o las migraciones interiores e internacionales, tanto en la actualidad como en los diversos contextos históricos. Así, estos conocimientos sin duda son una base muy sólida, científica, para mejor entender problemas tan espinosos como los relativos a la llegada e integración de los inmigrantes extranjeros en Europa, sobre todo en el contexto actual de dificultades en el mercado laboral; parecidos razonamientos se podrían hacer respecto a otros temas que preocupan a nuestra sociedad actual, como el envejecimiento demográfico, población y medio ambiente, población y ordenación del territorio, etc.

Unos conocimientos mínimos de geodemografía sin duda facilitan mucho la necesaria recepción crítica de las informaciones referidas a población que proporcionan los medios de comunicación, pues por la simplificación con que se ofrecen estas noticias, o por estar tamizadas políticamente, pueden derivar a interpretaciones inadecuadas o al menos insuficientes.

Como ejemplo de información geodemográfica insuficiente o errónea entre los españoles, baste citar el ejemplo de la presencia de extranjeros en España. Así, en 1995 según

4 Citado por A. ETCHELECOU, «Démographie et Aménagement du Territoire, un défi pour le développement durable», comunicación presentada a Xèm Colloque National de Démographie, «Démographie et Amenagement du Territoire», Bordeaux, 21-23 mai 1996 (mecanografiado).

5 GANI, L., «L'alphabétisation démographique», Populations et Societés, Paris, I.N.E.D., num. 277, 1993. 
datos oficiales había en España 500.000 extranjeros, equivalentes al 1'5 \% de la población censada en España en 1991; del total de extranjeros, son europeos el $51 \%$ y magrebíes el $16 \%$. Pues bien, según las encuestas realizadas en España en 1995 por el C.I.S. o por el C.I.R.E.S., el $68 \%$ de los españoles encuestados asocia extranjero con magrebí, y el $58 \%$ cree que el colectivo extranjero continental más numeroso en España es el magrebí, mientras sólo el $2 \%$ de los encuestados otorgan tal primacía a los extranjeros europeos ${ }^{6}$. Además, los españoles parecen bastante impactados y desorientados por el cúmulo de informaciones puntuales sobre los extranjeros en España, pues pese a lo reciente de esta inmigración y a su reducida cuantía absoluta y relativa -500.000 en 1995, 1'5\% de la población nacional-, el $30 \%$ de los españoles encuestados en 1995 cree que en España hay igual o más inmigrantes que en Francia (3’6 millones de inmigrantes en 1990, 6’3 \% de su población total) o que en Alemania (6’9 millones de inmigrantes en 1993, 8’5 \% de la población del país) ${ }^{7}$. Obviamente, estos errores sobre el número y nacionalidades de los extranjeros en España, no son algo anecdótico, sino que pueden ser de importancia a la hora de configurar las actitudes de los españoles hacia los extranjeros, y en concreto hacia los magrebíes, que son precisamente los extranjeros con incremento más rápido en nuestro país, especialmente los marroquíes, y también son los extranjeros que merecen valoración más baja a los españoles encuestados, al mismo tiempo que son percibidos como los extranjeros más problemáticos para su integración en España.

\section{Urbanización y población urbana: ejemplo de necesaria instrucción en geodemo- grafía}

Según manifiestan los geógrafos de la población más destacados de todos los países ${ }^{8}$, las preocupaciones centrales de la geografía de la población deben mostrar especial sensibilidad por los problemas que presentan las sociedades actuales; opiniones que en 1991 resumía Daniel Noin —Presidente de la Comisión de Geografía de la Población de la U.G.I.- con estas palabras: «... el optimismo [de la geografía de la población] se justifica plenamente si la sensibilidad a los problemas y a los interrogantes del mundo actual ..., se confirma en los próximos años... Los demogeógrafos no ganarán nada si eluden los grandes problemas de las sociedades o de la humanidad en este fin de siglo XX: estos problemas deben estar situados en el corazón de sus investigaciones».

Las fecundidades muy altas o muy bajas, las migraciones internacionales, y las redistribuciones espaciales de la población son sin duda temas mayores de nuestra disciplina en la actualidad, dadas sus repercusiones económicas, sociales, políticas o de ordenación del territorio, entre otras. Con ayuda de los medios de comunicación y de las preocupaciones políticas de los Gobiernos europeos occidentales, las dos primeras temáticas enunciadas se han popularizado más entre nosotros, es decir, la fecundidad baja asociada al envejecimiento, la fecundidad alta asociada a un crecimiento demográfico que puede ahogar el

6 GOZÁLVEZ PÉREZ, V., «La percepción del Mediterráneo a través de la inmigración: las actitudes de los españoles hacia los magrebíes», Investigaciones Geográficas, núm. 20, Instituto Universitario de Geografía de la Universidad de Alicante, 1998, pp. 5-18.

7 S.O.P.E.M.I., Tendances des migrations internationales. Rapport annuel 1994, O.C.D.E., Paris, 1995, $252 \mathrm{pp}$.

8 NOIN, D., Où va la géographie de la population?, U.G.I., Commission de Géographie de la Population, 1991, $44 \mathrm{pp}$. 
desarrollo económico, y las migraciones internacionales asociadas tanto a las dificultades de creación de empleo como a los problemas de xenofobia e integración en los países occidentales receptores de inmigrantes. Sin embargo, las redistribuciones espaciales de la población, asociadas al éxodo rural, a la concentración urbana y a la ordenación del territorio, aún siendo aparentemente menos «dramáticas», no por ello tienen menos interés actual para el geógrafo de la población, bien sea a escala planetaria, a escala nacional o a escala profundamente local.

La urbanización demográfica, aunque es un aspecto intrínseco al desarrollo económico y social de cualquier país, en los países en desarrollo tal proceso se realiza durante los últimos decenios con excesiva celeridad, de modo que se le reconoce como un grave problema, y así es calificado expresamente en las Resoluciones aprobadas por la Conferencia Internacional sobre Población y Desarrollo celebrada en El Cairo en 1994 (Resolución X). De hecho, manuales recientes destinados a nuestros estudiantes universitarios de los primeros cursos, destacan la concentración demográfica en las aglomeraciones urbanas como uno de los grandes problemas actuales de la población ${ }^{9}$.

Tal como se repite en manuales, estudios o en las fuentes estadísticas, sobre todo en las internacionales ${ }^{10}$, la definición de población urbana es, hoy por hoy, un problema que tiene muy lejos el consenso internacional. Así, el Anuario Demográfico de Naciones Unidas continúa respetando las muy dispares definiciones de población urbana que ofrecen los censos de población de cada país, pues «la distinción entre regiones urbanas y rurales está tan estrechamente unida a consideraciones de orden histórico, político, cultural y administrativo, que sólo se puede progresar lentamente hacia definiciones y métodos uniformes» (edición 1995, p.44). Así, sin salir de Europa, en Albania es población urbana la que reside en localidades con más de 400 habitantes, en Austria se requiere que el municipio tenga más de 5.000 habitantes, en Bulgaria son localidades urbanas las que poseen tal status jurídico, con independencia del número de habitantes, aunque el umbral más repetido en Europa para la calificación de población urbana es la de la aglomeración con más de 1.500 ó 2.000 habitantes, bien que acompañados de muy diversas casuísticas que hacen referencia a la distancia entre edificios, a las migraciones diarias intermunicipales por motivos de trabajo, o a la proporción de activos no agrícolas.

En el caso de España, las estadísticas de población urbana aún están lejos de una solución satisfactoria. Por motivos operativos, en las investigaciones suele utilizarse el criterio de municipios con más de 10.000 habitantes, aunque los censos de población en 1950 introdujeron el criterio de «entidad de población» con más de 10.000 habitantes para la calificación de la población como urbana, con lo que se rebaja considerablemente la tasa de población urbana: así, en 1981 con el criterio municipio de más de 10.000 habitantes era población urbana el 73`2 \% del total, mientras con el criterio «entidad con más de 10.000 habitantes» sólo lo era el 628 \% ${ }^{11}$. Por su parte, en el Anuario Demográfico de

9 VÉRON, J., Démographie, Paris, Armand Colin, Coll. U flash, 1991, 128 pp.

PUYOL ANTOLÍN, R.; VINUESA ANGULO, J.; ABELLÁN GARCÍA, A., Los grandes problemas actuales de la población, Madrid, Ed. Síntesis, 1993, 235 pp.

10 NOIN, D., Géographie de la population, Paris, Armand Colin, 1994 (4ª edit.), 280 pp. THUMERELLE, P.J., Las poblaciones del Mundo, Madrid, Ed. Cátedra, 1997, 427 pp. GOZÁLVEZ PÉREZ, V., «Los procesos de urbanización: siglos XIX y XX», ap. Los procesos de urbanización; siglos XIX y XX, Alicante, Instituto de Cultura Juan Gil-Albert; Seminari d’Estudis sobre la població del País Valencià, 1991, pp. 9-23. United Nations, Demographic Yearbook. Para este trabajo se han consultado las ediciones de 1987 y 1995.

11 GOZÁLVEZ PÉREZ, V., «Los procesos de urbanización ....», Ob.cit. 
Naciones Unidas, para la población del Censo de población de España de 1981 se cita como urbana el $91 \%$ del total (edición de 1987), que corresponde a la población que reside en municipios con más de 2.000 habitantes, mientras para la población del Censo de 1991 la tasa de población urbana se rebaja al 64 \% (edición de 1995), que corresponde a la que reside en entidades de población con más de 10.000 habitantes.

A estas variaciones de criterios, el Censo de población de España de 1991, ha añadido un nuevo hecho que sin duda resulta negativo para nuestro progreso estadístico en la calificación de la población urbana. En efecto, la modificación introducida en el Censo de 1950 referida a entidades de población, sin duda fue muy positiva para avanzar en una mejor delimitación de población urbana sobre el criterio municipio, dada la gran variación de superficie y de poblamiento que los municipios tienen en España. El criterio del poblamiento introducido en el Censo de 1950 se podría haber perfeccionado con la nueva variable que aparece en el Censo de población de 1981 que hacía referencia al municipio de trabajo o de estudio respecto al de residencia, los desplazamientos que tales actividades originan, los medios de transporte utilizados y el tiempo que se tarda en tales desplazamientos; sin embargo, estas informaciones estadísticas han sido suprimidas en el Censo de población de España de 1991'2. Frente a esta supresión del Censo de 1991, los desplazamientos intermunicipales por motivos de trabajo son considerados criterio básico en otros países europeos para delimitar las áreas urbanas supramunicipales o las cuencas de empleo, delimitaciones espaciales ambas de evidente interés para la ordenación del territorio $^{13}$. En efecto, la actual movilidad entre lugar de residencia y lugar de trabajo, que han incentivado la motorización y la especialización en los usos del suelo, ya han dejado insuficientes los criterios de entidad de población y de municipio de residencia para establecer una calificación adecuada de población urbana en España.

Con las reservas indicadas sobre las estadísticas internacionales, la tasa de población urbana de los países menos desarrollados era del $17 \%$ en 1950, mientras en 1995 ya alcanza al 41\%, lo que representa poblaciones absolutas de 294 millones de habitantes en la primera fecha, y 1.868 millones en la segunda ${ }^{14}$, es decir, durante estos 45 años la población urbana de los países menos desarrollados ha crecido a razón de 4`2 \% anual. De inmediato resalta, pues, las dificultades que este acelerado y sostenido crecimiento de la población urbana provoca en las infraestructuras urbanas de estos países o en sus servicios sociales.

A efectos educativos, convendría resaltar no sólo las dificultades que puede originar el proceso de urbanización en sí mismo - resultado conjuntamente del éxodo rural, del propio crecimiento natural de la población urbana y de los cambios de criterios territoriales sobre población urbana que se producen en cualquier país con el paso del tiempo-, sino también hay que hacer notar sus aspectos positivos, como es el facilitar la disminución de la fecundidad excesiva en relación al desarrollo económico. Así, para el caso de Marruecos se señala que sus ciudades, pese a todas las carencias, son centros de desarrollo relativo respecto a las áreas rurales, lo que en términos de comportamiento demográfico, se traduce en un descenso acusado de su fecundidad, a resultas de mayores posibilidades y

12 I.N.E., Censos de Población y Viviendas 1991. Metodología, Madrid, 1994, p. 83.

13 LE GLEAU, J.P., «Zonages fonctionnels pour le développement local en France», X Colloque National de Démographie, «Démographie et Aménagement du Territoire», Bordeaux, 21-23 mai 1996, 17 pp. (mecanografiado).

14 THUMERELLE, P.J., Ob.cit. 
efectividad en su planificación familiar. Esto último, a su vez está conexionado, entre otros factores, con mayores asistencias sanitarias en las ciudades, un aumento de la instrucción femenina o un matrimonio más tardío ${ }^{15}$ : así, en 1990-94 el número de hijos por mujer en Marruecos era de 2’2 en las ciudades $-51^{\prime} 5 \%$ de población urbana en 1994 - y de 4’5 hijos por mujer en el medio rural.

Otra consecuencia de la urbanización acelerada en los países menos desarrollados, es la alimentación de su emigración exterior subsiguiente al éxodo rural interior. Es decir, la masificación del éxodo rural hacia las ciudades, supone trasladar a éstas el subempleo y el paro de las zonas rurales sobrepobladas, mientras las posibilidades de empleo urbano son mínimas. Tales procesos son especialmente acusados en los países ribereños del sur del Mediterráneo, singularmente en Turquía y Marruecos ${ }^{16}$, los dos grandes emisores actuales de inmigrantes a Europa. Estos dos países siguen procesos demográficos y de urbanización extraordinariamente paralelos: sus deficiencias urbanas, junto a sus acelerados procesos de urbanización, crean fuertes motivaciones para la emigración exterior, que además, se ve fuertemente incentivada por las sólidas redes sociales formadas por familiares y amigos ya establecidos en Europa, donde se estima que actualmente residen unos 3 millones de turcos y entre 1 y 1'5 millones de marroquíes. Así vemos, que el proceso acelerado de urbanización en estos países surmediterráneos, junto con otros factores, produce otro hecho geodemográfico plenamente enmarcado en los procesos de globalización, y que afecta más o menos directamente a nuestra sociedad.

De un hecho geodemográfico con cuño de globalización, como son las migraciones internacionales incentivadas por la urbanización acelerada, pasamos a otro hecho geodemográfico urbano aparentemente opuesto, profundamente local, como es el estudio geodemográfico a escala de barrio urbano; escala de trabajo de especial actualidad y utilidad en los estudios geodemográficos, tal como manifiestan los atlas urbanos publicados desde los años ochenta en los países europeos, incluida España ${ }^{17}$. Sin embargo, y desde el punto de vista aplicado, además de la publicación aislada de atlas urbanos, quiero llamar la atención sobre la conveniencia de realizar atlas geodemográficos urbanos permanentemente renovados y concebidos como instrumentos de planificación; la dificultad está en los padrones municipales de habitantes, que deben ser la fuente idónea para la renovación permanente de estos atlas urbanos, y que en la última década se han empobrecido mucho en la información que recogen; no obstante, en la Comunidad Valenciana la información disponible a escala detallada del Censo de 1991, compensó, para esa fecha, las deficiencias del padrón. Sin embargo, es obvio que la frecuencia decenal de los censos limita el uso de sus datos con fines de ordenación del territorio. Así sería muy positivo conservar los padrones municipales de habitantes —y su renovación anual— con un número suficiente de variables, de modo que permitieran confeccionar cada cinco años este precioso

15 FADLOULLAH, A., «Transition urbaine, transition démographique et potential migratoire au Maroc», Conference méditerranéenne sur la population, les migrations et le développement. Actes. Tables rondes et Forum, Conseil de 1'Europe, Strasbourg, 1998, pp. 71-84.

16 FADLOULlAH, A., Ob.cit. IÇDUYGU, A., «Les migrations de le Turquie à destination de 1’Europe occidentale», Conference méditerranéenne sur la population, les migrations et le développement. Actes. Tables rondes et Forum, Conseil de 1’Europe, Strasbourg, 1998, pp. 23-40.

17 NOIN, D., Atlas des Parisiens, Paris, Masson, 1984, 180 pp. OCAÑA OCAÑA, Ma.C., Atlas social de la ciudad de Málaga, Málaga, Universidad, Instituto de Ciencias de la Educación, 1984, 314 pp. GOZÁLVEZ PÉREZ, V. y otros, Atlas sociodemográfico de la ciudad de Alicante, Alicante, Instituto de Estudios Juan GilAlbert de la Diputación Provincial; Ayuntamiento de Alicante, 1987, 143 pp. 
instrumento de planificación que son los atlas geodemográficos a escala de barrios. Obviamente, para ello los responsables políticos tendrían que estar convencidos de la utilidad de este instrumento para el gobierno municipal, lo que en muchos casos incluye, tal como decíamos al principio de nuestra intervención, una mayor formación geodemográfica en los ciudadanos, y sobre todo de los que tienen responsabilidades de gestión política.

\section{Conclusión}

Así, pues, como hemos visto de forma muy esquemática - y por supuesto incompleta- la geografía de la población es una disciplina con múltiples posibilidades educativas y aplicadas, y a todas las escalas, desde la mundial, con las múltiples interacciones que provocan los procesos de globalización, a la escala más profundamente local, como es el espacio geodemográfico de un barrio; parecidas utilidades se pueden enumerar desde el lado del «usuario» de la geografía demográfica, pues ésta es de interés para todo tipo de ciudadanos, desde los que tienen que utilizarla con fines de ordenación y gestión del territorio, a los que la utilizan para entender mejor las distintas sociedades, culturas o comportamientos demográficos.

Debemos, pues, hacer un esfuerzo, sobre todo los docentes e investigadores, para que la formación y las informaciones geodemográficas aumenten, tanto en sus contenidos científicos y pedagógicos, como con una difusión de la información científica mucho más amplia que la actual y más accesible a la comprensión adecuada de todos los ciudadanos. 